\title{
The financial cost of preventive and curative programs for breast cancer: a case study of women in Shiraz-Iran
}

\author{
Nahid Hatam ${ }^{1}$, Vahid Keshtkar ${ }^{2}$, Asiyeh Salehi ${ }^{3, *}$, Hamidreza Rafei $^{1}$
}

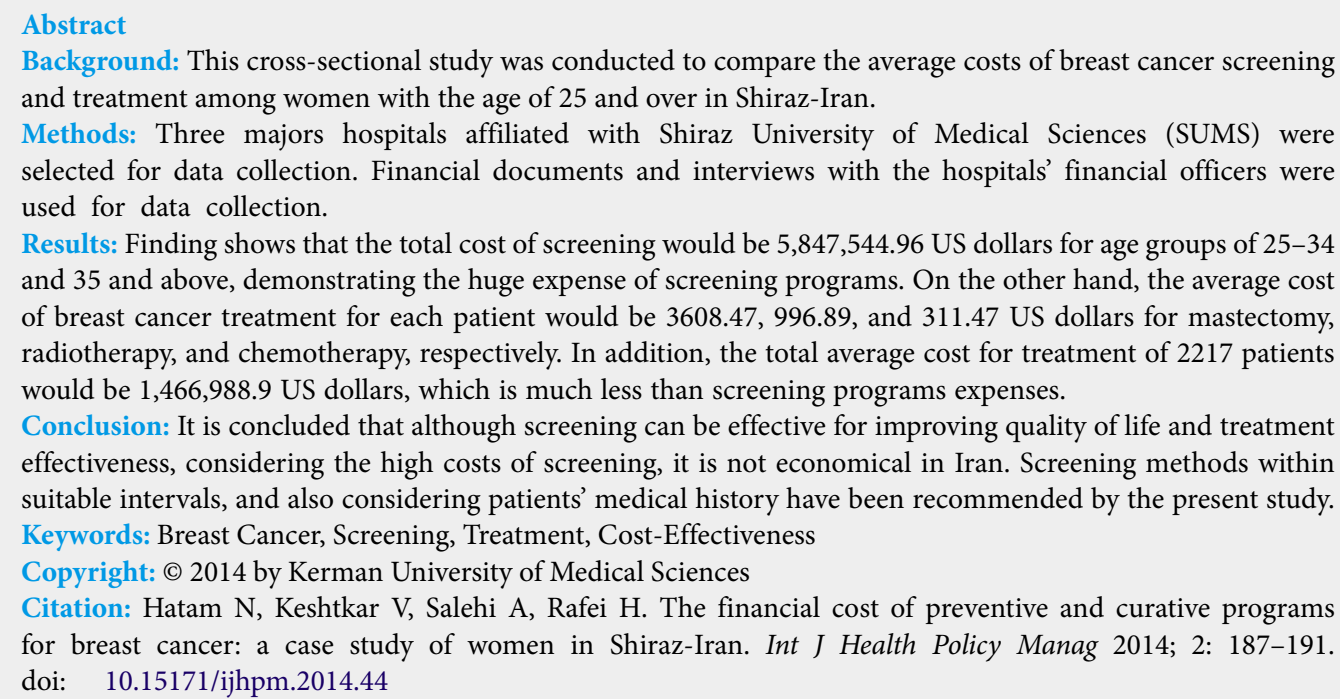

\section{Article History:}

Received: 14 January 2014

Accepted: 4 May 2014

ePublished: 8 May 2014

\section{Introduction}

Cancer is considered as a major issue concerning healthcare systems. Due to the global increases in cancer prevalence, and its attribution to more than $12 \%$ of the world's mortality, providing efficient policies for cancer screening and treatment is recognized as the leading priority of healthcare systems $(1,2)$. According to the literature, five common factors that contribute to increasing cancer rates in both developed and developing countries include dietary changes, smoking, infections, occupational and agronomic problems, and environmental pollution. Among these, dietary factors and infections alone contributed to 4.4 out of 10 million cancers in $2002(3,4)$.

In Iran, cancer is the third leading cause of mortality, following accidents and heart disease. 30,000 individual deaths are attributed to cancer annually. Additionally, Iran experiences more than 70,000 new cancer cases annually, and this figure could potentially double in the next two decades due an aging population (4).

Breast cancer is the most common cancer in women, and the second highest contributor to cancer mortality. It accounts for $20 \%$ of deaths in women aged 40 to 50 years (5), and $32 \%$ of women's cancers, and 19\% of women's cancer deaths, are attributed to breast cancer (6). Considering the current inconclusive knowledge regarding the nature of cancers, cancer-management solutions should focus on prevention, early detection, control, and cure (7).

According to World Health Organization (WHO) statistics, breast cancer prevalence rates presently increase by 1.8 to 2 yearly. Notably, $25 \%$ of all detected cases are in developed stages (5). Detection and preventive methods for breast cancer (breast sonography and mammography) are the most effective methods in the initial stages of breast cancer, otherwise increasingly complex treatments such as mastectomy, radiotherapy, and chemotherapy are utilised. However, such methods impose high costs for patients and hospitals (8). In developed countries, cancer screening is part of a national plan, which includes training for early detection, while in developing countries such as Iran, cancer screening is not yet institutionalized (8). A majority of cases in developed countries are detected and cured in stage 1, while in Iran many of cases are detected in the second or third stages, creating challenges for treatment (8).

According to the above information, breast cancer bears a high prevalence rate, and is an important social and health issue (9). However the presently increasing population creates a strain on available resources, particularly in developing countries, which necessitate optimized utilization of financial resources (9). Thus, it is necessary for healthcare service managers and specialists to computation technology for effective health services in order to optimize resource assignment (10). Considering breast cancer prevalence in the Fars province and the necessity of mammography and sonography, this study has been conducted to show the financial cost of mammography and sonography screening program for preventive and curative procedures among women with the age of 25 and over in Shiraz-Iran. 


\section{Methods}

This cross-sectional study has been conducted in 2007 (one year) in hospitals affiliated with Shiraz University of Medical Sciences (SUMS), including Shahid Faghihi, Hafez and Namazi hospitals. The study population includes two groups: 1) patients referred to Hafez hospital for sonography and mammography for breast cancer detection, and 2) patients referred to Shahid Faghihi hospital for mastectomy services, or to Namazi hospital for chemotherapy and radiotherapy services.

Among the patients referred to Hafez hospital, 225 were referred for sonography and 494 were referred for mammography services. 196 cases were referred to Shahid Faghihi hospital for mastectomy; 190 and 1831 to Namazi hospital for radiotherapy and chemotherapy, respectively. It should be noted that all cases were selected in the sample, due to the limited numbers of patients.

Data observation, financial documents and interviews with the hospitals' financial officers were used in data collection. Cost information was also based on the national tariff.

For estimating the at-risk population requiring sonography and mammography, data related to women aged 25-34 years and above 35 years, respectively, was extracted from the statistical report of the Fars governmental programming deputy. Costs were then computed and compared in order to clarify the resource assignment process. Two costs, namely those related to cancer prevention actions and costs related to treatment were compared in this study.

\section{Results}

Findings show that the average costs of breast sonography and mammography per patient were 3.13 and 10.98 US dollars respectively for the hospital (the average cost was estimated based on the prime cost; Table 1). The average expense of breast sonography and mammography incurred by each patient was 4.45 and 11.70 US dollars respectively. This shows that hospitals do not incur any additional costs for these services, as the cost is covered by patients.

Considering that mammography is the most suitable method for screening among women aged 35 years and over, sonography is generally used for patients under 35 years. Therefore, computations were completed in two different age groups to calculate the costs of screening, as follows:

- In accordance with annual statistics (2005), there were 265,627 women aged 25 to 34 years in Fars province. Assuming that they use sonography, at a cost of 3.14 US dollars per person, the total sonography cost would be 834,068.78 US dollars.

- In accordance with annual statistics (2007), there were 456,508 women above 35 years of age in Fars province. Considering that the cost of mammography is 10.98 US dollars, the total cost for mammography would be $5,012,457.84$ US dollars.

Therefore, the total cost of screening would be 5,845,490.67 US dollars for age groups of above 35 and 25-34 (Table 2), demonstrating the huge expense of screening programs. On the other hand, the average cost of breast cancer treatment for each patient is $3608.47,996.89$, and 311.47 US dollars for mastectomy, radiotherapy, and chemotherapy, respectively. The total average cost to treat 2217 patients would be $1,466,988.90$ US dollars (Table 3 ), which is much less than screening programs expenses.

\section{Discussion and Conclusion}

Cancer is considered as a global health imperative. It is predicted that cancer will become the leading cause of death by 2030 due to an aging population, relative control of infectious diseases, and increasing cancer risk factors such as pollution. Therefore providing efficient cancer-management techniques is essential in health systems (11).

Table 1. Costs of sonography and mammography for each patient in Hafez hospital in 2007

\begin{tabular}{|c|c|c|c|c|}
\hline \multirow{2}{*}{ Hafez hospital costs } & \multicolumn{2}{|c|}{ Costs of sonography } & \multicolumn{2}{|c|}{ Cost of mammography } \\
\hline & IRR & $\$$ & IRR & $\$$ \\
\hline Water cost & 33 & 0.00 & 464 & 0.04 \\
\hline Energy cost & 14 & 0.00 & 197 & 0.01 \\
\hline Natural gas coast & 18 & 0.00 & 255 & 0.02 \\
\hline Telephone cost & 8 & 0.00 & 116 & 0.01 \\
\hline Maintenance cost & 88 & 0.00 & 1232 & 0.12 \\
\hline Building depreciation cost & 330 & 0.03 & 4596 & 0.45 \\
\hline Security cost & 208 & 0.02 & 2909 & 0.29 \\
\hline Transportation cost & 80 & 0.00 & 1123 & 0.11 \\
\hline Cleaning services cost & 485 & 0.04 & 6777 & 0.67 \\
\hline Data processing cost & 121 & 0.01 & 1751 & 0.17 \\
\hline Waste disposal cost & 9 & 0.00 & 123 & 0.01 \\
\hline Consumption material costs & 5602 & 0.56 & 18365 & 1.83 \\
\hline Personnel cost & 19959 & 1.99 & 45617 & 4.56 \\
\hline Staff uniform clothes cost & 196 & 0.01 & 2210 & 0.22 \\
\hline Cost of equipment depreciation & 4210 & 0.42 & 24110 & 2.41 \\
\hline Total cost & 31361 & 3.13 & 109845 & 10.98 \\
\hline
\end{tabular}


Table 2. Costs of breast cancer screening method in Fars province in 2007

\begin{tabular}{|c|c|c|c|c|c|}
\hline \multirow{2}{*}{ Diagnostic services } & \multirow{2}{*}{$\begin{array}{l}\text { The study population (on the basis of } \\
\text { Fars province statistical calendar in 2005) }\end{array}$} & \multicolumn{2}{|c|}{ The prime cost per each patient } & \multicolumn{2}{|c|}{ The total cost for breast cancer screening } \\
\hline & & IRR & $\$$ & IRR & $\$$ \\
\hline Breast sonography & 265627 (age: 25-34) & 31361 & 3.14 & $8,340,687,847$ & $834,068.78$ \\
\hline Mammography & 456508 (age: $35+$ ) & 109845 & 10.98 & $50,124,578,460$ & $5,012,457.84$ \\
\hline
\end{tabular}

Table 3. Costs of treatment methods in under study population in 2007

\begin{tabular}{|c|c|c|c|c|c|}
\hline \multirow{2}{*}{ Treatment services } & \multirow{2}{*}{ Number } & \multicolumn{2}{|c|}{ The average of payment per patient } & \multicolumn{2}{|c|}{ The patient payment per year } \\
\hline & & IRR & $\$$ & IRR & $\$$ \\
\hline Radiotherapy & 190 & 9968925 & 996.89 & $1,894,095,780$ & $189,409.57$ \\
\hline Mastectomy & 196 & 36084790 & 3608.47 & $7,072,618,992$ & $707,261.89$ \\
\hline Chemotherapy & 1831 & 3114796 & 311.47 & $5,703,191,490$ & $570,319.14$ \\
\hline
\end{tabular}

Breast cancer is one of the most prevalent cancers worldwide. Although breast cancer is common in both developed and developing countries, its prevalence is increasing in the developing world due to increased life expectancy, increased urbanization, and adoption of western lifestyles (5). In addition, these countries experience high case fatality rates, likely due to a lack of awareness of the benefits of detection and treatment, a scarcity of adequate facilities for detection and diagnosis, as well as poor access to primary treatment (5). Therefore, the necessity of using screening methods such as sonography and mammography for detecting and preventing this cancer is evident (12).

The importance of detecting breast cancer during the first stages has been highlighted by many studies. For example Anderson et al. emphasized on the role of mammography in reducing cancer returns (13). Zwahlen et al. revealed that breast cancer patients would have a greater chance of survival if the disease is detected in its initial stages (14). Therefore, early detection is an important step towards reducing the physical and mental impacts of breast cancer. Evidence shows that general systematic invitations to breast imaging, for women aged 50-69 years, can reduce breast cancer risk up to $25 \%(14,15)$.

In addition to planned screening, the quality and duration of screening are also important prevention/detection factors. Anderson et al. stated that systematic breast imaging every two years can help to detect the disease in its initial stages (13). Some evidence suggests that regular screening can reduce the risk of breast cancer by $30 \%$ in women aged over 50 years, as early detection allows more effective treatment, at lower costs (13). Lebovic et al. emphasized the importance of screening in the USA (16). Pisati et al. also examined 417 under X-ray employees during 2002-9 with cancer screening methods, in which the participation rate, the premalignant and malignant findings, and cost-effectiveness are consistent with the suggestion that cancer screening programs can be implemented as health promotion activities in healthcare workers (17).

According to the statistics, the prevalence rate of breast cancer in Iran's Fars province is 6.7 per 1000 for women aged over 35 years (456,508 individuals) (18), which shows an estimation of 3058 individuals. Assuming an average treatment cost of 661.70 US dollars per patient, the total cost for treating afflicted individuals will be 2,023,478.60 US dollars. Compared with annual mammography screening costs, calculated to be 2,991,031.38 US dollars in the previous section, these figures suggest cost-ineffectiveness of screening in women aged over 35 years. Grann et al. also suggested that surgery is more cost-effective than screening, as annual mammography imposes high costs (19), which is consistent with our findings. However some of the studies such as Smith and Zwahlen et al. emphasized on the effectiveness of screening methods, while there is a good insurance coverage for that $(14,20)$.

Breast cancer prevalence for women aged 25-34 years was 9.16 per 100,000 in Fars province. According to Mehrabani et al. study in 2008 (21), the expected breast cancer cases in this age group $(n=265,627)$ is 24.33 . Therefore, considering the costs of screening using sonography $(833,032.83$ US dollars), and the expected cases' treatment costs $(16,099.17$ US dollars), sonographic screening is shown to be ineffective in comparison with treatment (816,933.65 US dollars). This reveals that sonography, like mammography, requires more funding than treatment. Therefore, it appears that screening is not economical for this sample population, and it is recommended to perform screening in accordance with patients' health history and age. Thus, in this study, screening was performed with different time interventions such as 2 or 3 years and the obtained results suggested that:

- Assuming screening with mammography takes place every 2 years in Fars province, the total costs of breast cancer screening for the above 35 years age group will be 5,014,512.12 US dollars. Comparing this value with treatment costs of 4,046,961.48 US dollars shows the cost-ineffectiveness of screening methods about 967,550.64 US dollars.

- Assuming screening with sonography takes place every 2 years in Fars province, the total costs of breast cancer screening for women aged between 25-34 years group will be $833,032.38$ US dollars. Comparing this value with treatment costs of $32,198.35$ US dollars shows the cost-ineffectiveness of screening methods 800,834.47 US dollars.

- Assuming screening with mammography every 3 years in Fars province, the total cost of breast cancer screening for the above 35 years age group will be 6,070,442.22 US 
dollars, which shows higher efficiency of 1,055,930 US dollars in compare with treatment.

- For sonography in women aged 25-34 years in Fars province, the findings also showed that the sonography cost with interval of three years is $833,032.38$ US dollars which is not cost-effective with the amount of $784,728.23$ US dollars in comparison with treatment cost $(48,304.15$ US dollars). Therefore it appears that screening is not effective for 25-34 year old group, even if when performed every three years.

Consistent with these findings, other studies also revealed that yearly screening is not cost-effective. For example, Schousboe et al. studied breast cancer screening using mammography for 40-50 year old women in California, and concluded that the cost of this plan is lower than 100,000 US dollars per Quality Adjusted Life Year (QALY) for women aged 40-79 years. The cost of 40-49 year old women was 50000 US dollars per QALY. This shows that annual mammography is not costeffective for all age groups. Thus, the general consensus is that mammography should be performed based on age, breast density, breast biopsy history, and family history (22). Boer et al. also studied the effectiveness of decreasing the imaging interval from 3 years to 2 years, however with increasing the average age from 64 years to 69 in Britain. They concluded that both of these changes increase survival and enhance the health efficiency (23). On the other hand, Stout et al. study revealed that the death rate due to breast cancer has gradually decreased in the USA in recent decades, partly due to disciplined screening. This study suggested that in 2001, about $70 \%$ of American women had performed at least one screening in last two years. This study showed the costeffectiveness of breast cancer imaging in 1-2 year intervals for women aged over 40 years (24), which is not consistent with our results, and may be due to different healthcare systems in the US and Iran.

Peregrino et al. also considered screening methods such as mammography and other methods per 2-year intervals as a default. He has studied four scenarios, namely disease history, mammography, digital mammography, and imaging and used Markov's biannual model for 100,000 individuals. Although results suggested that these methods have different costs, and the difference of effectiveness was between 300 to 78,000 total years of survival, it was concluded that mammography is the most cost-effective scenario (25).

Although many of studies reveal that the screening methods are very useful for detecting and preventing diseases, especially breast cancer, they emphasize that some factors such as screening interval, target population, age, and detection method are important for cost-effectiveness of cancer treatment methods. For example Verbeek et al. studied 5 kinds of cancer such as breast, cervical, colorectal, prostate, and lung, and uncovered some serious problems associated with screening, such as unnecessary referrals for certain detection in hospital (26).

It is concluded that screening especially for women aged over 40 years can be considered an effective tool for improving quality of life and treatment effectiveness. However, considering the high costs of screening, it is not economical in most countries.
Limitations

One of the limitations of the present study is that the cost of screening programs is not certain and precise as sensitivity, specificity and negative and positive predictive value of screening programs (mammography and sonography) which could affect the overall cost was not calculated in this study. The second limitation is that the costs of breast cancer occur over time (during several years). Therefore looking at only one year might not indicate true cost of treatment.

Ethical issues

Ethical approval for this study was granted by the human research ethics committee of Shiraz University of Medical Sciences.

Competing interests

Authors declare that they have no competing interests.

\section{Authors' contributions}

$\mathrm{NH}$ and $\mathrm{AS}$ contributed to writing introduction, writing findings and discussion. $\mathrm{VK}$ and $\mathrm{NH}$ contributed to designing the study. HR had a substantial role in collecting data and analysis.

\section{Authors' affiliations}

${ }^{1}$ School of Health Administration and Medical Information, Shiraz University of Medical Sciences, Shiraz, Iran. ${ }^{2}$ Department of Community Medicine, Shiraz University of Medical Sciences, Shiraz, Iran. ${ }^{3}$ Griffith Health Institute, Griffith University, QLD, Australia.

\section{References}

1. Micheli A, Coebergh JW, Mugno E, Massimiliani E, Sant M, Oberaigner W, et al. European heath systems and cancer care. Ann Oncol 2003; 14: 41-60.

2. Mohaghegh FA, Hamta A, Shariat Zade SA. [Investigation of current cancers in Markazi province and its registration system in 2001-2006 in compare with country statistics]. Journal of Arak University of Medical Sciences 2008; 11: 84-94.

3. Akbari ME, Naghavi M. [Economical burden of cancers in Iran]. Daroo and darman Journal 2009; 67: 51-6.

4. Hatam N, Ahmadloo N, Ahmad Kia Daliri A, Bastani P, Askarian M. Quality of life and toxicity in breast cancer patients using adjuvant TAC (docetaxel, doxorubicin, cyclophosphamide), in comparison with FAC (doxorubicin, cyclophosphamide, 5-fluorouracil). Arch Gynecol Obstet 2011; 284: 215-20. doi: 10.1007/s00404-010-1609-8

5. Yen JY, Ko CH, Yen CF, Yang MJ, Wu CY, Juan CH, et al. Quality of life, depression, and stress in breast cancer women outpatients receiving active therapy in Taiwan. Psychiatry Clin Neurosci 2006; 60: 147-53. doi: 10.1111/j.1440-1819.2006.01479.x

6. Bland KI, Sarr MG, Buchler MW, Csendes A, Garden OJ, Wong J. General Surgery: Principles and International Practice. Australia: Springer; 2010.

7. Phipps WJ, Long BC, Woods N. Medical-surgical nursing: concepts and clinical practice. 5th ed. St. Louis, MO: Mosby; 1995.

8. Mousavi SM, Montazeri A, Mohagheghi MA, Jarrahi AM, Harirchi I, Najafi M, et al. Breast Cancer in Iran: an epidemiological review. Breast J 2007; 13; 383-91. doi: 10.1111/j.15244741.2007.00446.x

9. Gholikhani R, Sadrmomtaz N. [Health cost analysis]. 1st ed. Tehran: Debizesh publication; 2001.

10. Nouroozi T, Salehi A. Prime costs of clinical laboratory services in Tehran Valiasr Hospital in 2009. EMHJ 2013; 19: 159-65.

11. Hatam N, Bastani P, Ahmadloo N, Kiadaliri AA. Health Related Quality of Life in Breast Cancer Patients Undergoing Current Chemotherapy Protocols. Kerman University of Medical 
Sciences Journal 2011; 19: 37-46.

12. Phipps WJ, Long BC, Woods N. Medical-surgical nursing. 7th ed. St. Louis, MO: Mosby; 2003.

13. Andersen R, Hager M. Analysis of the cost-effectiveness of mammography promotion by volunteers in rural communities. Health Educ Behav 2002; 29: 755-70. doi: 10.1177/109019802237942

14. Zwahlen M, Probst N, Baschung B, de Wolf C, MartyTschumi E, Borisch B. Early detection of breast cancer using mammography-a position paper of the Swiss Cancer League [internet]. 2003. Swiss Cancer League. Available from: http:// assets.krebsliga.ch/downloads/pospap_final_kls_en_.pdf

15. Risenquist J, Lindfors K. Screening mammography in women aged 40-49 years: analysis if cost-effectiveness. Radiology 1994; 191: 647-50.

16. Lebovic GS, Hollingsworth A, Feig SA. Risk assessment, screening and prevention of breast cancer: A look at costeffectiveness. Breast 2010; 19: 260-7. doi: 10.1016/j. breast.2010.03.013

17. Pisati G, Cerri S, Marinelli M, Tedeschi B, Valsecchi E. [Cancer screening programme in health care workers]. G Ital Med Lav Ergon 2011; 33: 57-60.

18. Hadi N, Sadeghi-Hassanabadi A, Talei AR, Arasteh MM, Kazerooni T. Assessment of a breast cancer screening programme in Shiraz, Islamic Republic of Iran. East Mediterr Health J 2002; 8: 386-92.

19. Grann VR, Patel PR, Jacobson JS, Warner E, Heitjan DF, AshbyThompson $\mathrm{M}$, et al. Comparative effectiveness of screening and prevention strategies among BRCA1/2-affected mutation carriers. Breast Cancer Res Treat 2011; 125: 837-47. doi: 10.1007/s10549-010-1043-4

20. Smith RA. Cancer screening in the USA. J Med Screen 2006; 13: 48-53.

21. Mehrabani D, Tabei SZ, Heydari ST, Shamsina SJ, Shokrpour N, Amini M, et al. Cancer Occurrence in Fars Province, Southern Iran. Iran Red Crescent Med J 2008; 10: 314-22.

22. Schousboe JT, Kerlikowske K, Loh A, Cummings SR. Personalizing mammography by breast density and other risk factors for breast cancer: analysis of health benefits and cost-effectiveness. Ann Intern Med 2011; 155: 10-20. doi: 10.7326/0003-4819-155-1-201107050-00003

23. Boer $R$, de Koning $H$, Threlfall $A$, Warmerdam $P$, Street $A$, Friedman $\mathrm{E}$, et al. Cost effectiveness of shortening screening interval in extending age range of NHS breast screening programme: computer simulation study. BMJ 1998; 317: 376-9. doi: $\quad 10.1136 / \mathrm{bmj} .317 .7155 .376$

24. Stout NK, Rosenberg MA, Trentham-Dietz A, Smith MA, Robinson SM, Fryback DG. Retrospective cost-effectiveness analysis of screening mammography. J Natl Cancer Inst 2006; 98: 774-82. doi: 10.1093/jnci/djj210

25. Peregrino AA, Vianna CM, de Almeida CE, Gonzáles GB, Machado SC, Costa e Silva FV, et al. [Analysis of Costeffectiveness of screening for breast cancer with conventional mammography, digital and magnetic resonance imaging]. Cien Saude Colet 2012; 17: 215-22.

26. Verbeek AL, van Dijck JA, Kiemeney LA, Broeders MJ. Responsible cancer screening. Ned Tijdschr Geneeskd 2011; 155: A3934.

\section{Key Messages}

Implications for policy makers

In order to provide more cost-efficient services it is recommended to:

- Perform screening methods within suitable intervals, and

- Paying attention to the patients' medical history in order to help the necessity of screening programs.

\section{Implications for public}

In order to decrease the unnecessary referrals for breast cancer screening it is recommended to consider age and medical history. The necessity of breast cancer screening increases with the age particularly more than 40 and also individuals with high risk medical history. These systematic referrals could improve the cost-efficient of healthcare services for both patients and healthcare providers. 